International Braz J Urol

Vol. 34 (3): 270-276, May - June, 2008

\title{
Fluoroscopy Guided Instillation Therapy in Chyluria Using Combination of Povidone Iodine with Contrast Agent. Is a Single Instillation Sufficient?
}

\author{
Gyanendra Sharma, Vinayak Chitale, Rajgopal Karva, Anshu Sharma, Abdul Bari Durug
}

Chitale Clinic Pvt. Ltd, Solapur, Maharashtra, India

\begin{abstract}
Purpose: To evaluate the safety and efficacy of a single instillation in a combination of povidone iodine with contrast agent under fluoroscopy guidance for the treatment of chyluria.

Materials and Methods: From December 1999 to July 2006 a total of 40 patients with chyluria were treated by renal pelvic instillation therapy (RPIS). The sclerosing solution was prepared using povidone iodine with contrast agent diluted with sterile water in a ratio of 1:1:3. It was instilled on the side having chylous efflux using a bulb tip ureteric catheter. Unilateral instillation was done in 26 cases, 10 on the right side and 16 on left. Fourteen patients had bilateral chylous efflux and RPIS was performed on both sides in the same session. Fluoroscopy was used to evaluate the complete filling of the pelvic calyceal system. The sclerosing solution was kept in the system for 5 minutes and the ureteric catheter was then withdrawn.

Results: Immediate clearance was observed in 39 patients. Recurrence occurred in five patients. They were treated again using the same procedure with satisfactory results. The longest follow-up was five years and the shortest five months.

Conclusion: RPIS of chyluria using a single instillation a combination of povidone iodine with contrast agent is safe and effective. Use of fluoroscopy helps to determine the exact amount of sclerosing solution required to completely fill the system and therefore overfilling is avoided. Moreover, the complications, which arise due to pyelointerstitial backflow, are prevented.
\end{abstract}

Key words: chyle; chyluria; povidone iodine

Int Braz J Urol. 2008; 34: 270-6

\section{INTRODUCTION}

Chyluria is basically a urological manifestation of lymphatic system disease, occurring as a result of communication between the lymphatics and renal pelvis (1). Although not life threatening it often causes morbidity due to its presentation like hematochyluria, colics, etc. It also leads to nutritional deficiency and a state of compensated immunosuppression (2). Chyluria is a condition with spontaneous remissions and exacerbations. Treatment with high protein and low fat diet is effective in some patients whereas anti- filarial drugs are not helpful in this late manifestation of parasitic infestation by Wuchereria bancrofti (3). The treatment most frequently used is renal pelvic instillation sclerotherapy (RPIS) to cause sclerosis of pyelo-lymphatic fistulae. Various sclerosants like silver nitrate in varying concentrations of $0-1 \%-3 \%$, povidone iodine $0.2 \%$, sodium iodide $15-25 \%$, potassium bromide $10-25 \%$, dextrose $50 \%$, hypertonic saline and meglumine diatrizoate (Urograffin76\% Schering Pharm, Germany) have been used (4-10).

Although silver nitrate enjoys wide popularity and has a success rate of $68-80 \%$, its preparation 
involves several steps subject to human error. It can also be associated with side effects like flank pain, nausea, vomiting, interstitial nephritis, chemical cystitis, papillary necrosis, arterial hemorrhage, pelviocalyceal cast formation, ureteric strictures, acute renal failure. Moreover, even death has been reported with its use $(4,10-14)$.

In the search to obtain an efficacious but less toxic and safe alternative povidone iodine has been used (7-9). It has been used either as a single instillation of $8-10 \mathrm{~mL}$ of diluted solution (7) or as 8 hourly instillation of total 9 doses (8) or in combination with $50 \%$ dextrose twice a day for 3 days (9).

We have studied the combination of povidone iodine with contrast agent as a single instillation using fluoroscopy to determine the exact amount of sclerosing solution needed.

\section{MATERIALS AND METHODS}

From December 1999 to July 2006, 40 patients (24 males, 16 females) were treated for chyluria. The majority of patients were in the 20-30 year age group (Table-1). All patients presented with a previous history of passage of milky white urine. The duration of symptoms ranged from eight years to four months. The associated symptoms were hematuria in 17, flank pain in five, dysuria in three, fever in three and passage of chylous clots in 11 patients. One patient had previously undergone RPIS using silver nitrate but had no relief from symptoms.

The diagnosis of chyluria was made by the ether test in all patients. Abdominal ultrasound was done as part of the protocol in all patients. It did not show any abnormality in any patient. Intravenous

Table 1 - Age distribution.

\begin{tabular}{lc}
\hline Age (years) & N of Patients \\
\hline$<20$ & 1 \\
$20-30$ & 20 \\
$30-40$ & 14 \\
$>40$ & 5 \\
\hline
\end{tabular}

urography was carried-out in the first 12 patients and was essentially normal. Pyelo-lymphatic communication was not observed in any of the patients on intravenous urography. Sixteen patients had previously received a course of diethylcarbamazine. Those who had not received the course were started on diethylcarbamazine.

All patients underwent cystoscopy under general anesthesia. They were asked to include some butter in their meal the night before the procedure. This was very helpful in localizing the side of chylous efflux. In 26 cases the chylous efflux was unilateral; 10 on the right side and 16 on the left side. The efflux was observed bilaterally in 14 patients. All the sides showing chylous efflux were subjected to RPIS.

The sclerosant solution was prepared by using povidone iodine $5 \%$, contrast agent (Urograffin $76 \%$, Schering Pharm, Germany) and sterile water in the ratio of 1:1:3. A bulb tip (Chevassu) ureteric catheter was used to instill the sclerosant in the pelviocalyceal system. Imaging in the form of C-arm fluoroscopy was used in all patients to visualize the complete filling of the pelviocalyceal system. The system was filled until blunting of all fornices was seen. Thus, over distension of the system and the consequent risk of pyelointerstitial backflow of the sclerosing solution was avoided. The ureteric catheter was kept at the ureteric orifice to prevent the sclerosant from being drained in the bladder. The other end of the ureteric catheter was blocked to prevent the sclerosant from dripping out. The sclerosant remained in the system for five minutes and then the ureteric catheter was removed.

In patients with bilateral chylous efflux both sides were treated in the same session.

In the course of follow up serum creatinine was evaluated in those who had undergone bilateral RPIS. Intravenous urography was done after one month in the first five patients but was not done in the subsequent patients.

\section{RESULTS}

Of the 40 patients, all, except one patient, had immediate clearance of urine. In one patient the chyluria persisted for two days after RPIS but subse- 
quently the chyluria stopped and the patient was free from symptoms at a follow up of five months.

There was recurrence of chyluria in five patients. One had recurrence after one month, two patients had recurrence after three months and another two patients had recurrence after six months. All were retreated using the same procedure as described above. No relapse was noted after re-treatment.

The patient who had a previous RPIS using silver nitrate did not show any recurrence after RPIS using povidone iodine with contrast agent.

The post treatment period was uneventful in all patients except for minimal pain and dysuria in some cases. Post treatment intravenous urography was done in only in five patients. Three of them had undergone bilateral RPIS. It was found to be normal in all patients. In view of this finding and the minimal theoretical risk of renal damage or ureteric strictures using this technique and sclerosing agent, subsequent patients were not subjected to intravenous urography. Serum creatinine was normal in the follow-up studies of all patients who had undergone bilateral RPIS.

None of the patients, except the five mentioned above, had recurrence during the follow up. This was confirmed by examining the urine by the ether test. The longest follow-up was five years and the shortest was five months. The average follow-up is one year.

\section{COMMENTS}

Chyluria usually affects the lower socio-economic class and is not uncommon in India, China, Japan, Taiwan, Africa or in South East Asian countries (15). Although a variety of parasitic and non-parasitic causes can cause chyluria, it is generally agreed that it should be considered as filarial unless proved otherwise, particularly in areas where lymphatic filariasis is or was endemic (16). Non-parasitic causes such as malignant tumors of the thoracic duct, pregnancy, trauma etc. are rare (6).

Parasitic infection causes obstruction to the retroperitoneal lymphatics leading to dilatation, proliferation and subsequent rupture of the lymphatics into the pelviocalyceal system (15). Recent observations suggest that the extensive lymphangiectasia observed in Bancroftian filariasis is secondary to lymphatic dysfunction caused by cytokines liberated by adult filarial worms and by the host immune responses to the parasite $(17,18)$.

The diagnosis of chyluria can be made by observing the urine sample and by doing the ether test $(4,6)$. Goel et al. found lymphocyturia a more sensitive tool to diagnose chyluria than the ether test (8). In the present study all patients with suspected chyluria had a positive ether test. Lymphocyturia was not evaluated in any of the patients. Additional tests to localize the pyelo-lymphatic communications, like lymphangiography and Intravenous Urography, are neither found to be useful nor cost effective $(4,5)$. We did not carryout a lymphangiography in any of the patients. Those patients who had an IVU did not show any pyelo-lymphatic communication.

Chyluria is debilitating and causes morbidity but is not life threatening. Hence the treatment of chyluria should be safe, effective and minimally invasive. RPIS has been considered the most popular form of treatment. The basic principle is to instill a sclerosant in the renal pelvis so that it can enter the pyelo-lymphatic communications and induce an inflammatory reaction. This chemical lymphangitis leads to edema of the lymphatic channels and the resultant blockage leads to immediate relief. Subsequent healing by fibrosis results in permanent remission (4).

The most commonly used sclerosant is silver nitrate. It has been used in concentrations ranging from $0.1 \%$ to $3 \%$ and instilled using varying protocols ranging from a single instillation up to as many as nine instillations over 3 days $(4-6,19)$. Dalela et al. have found that three instillations performed every eight hours in a single day are as effective and associated with less morbidity as compared to nine instillations done every eight hours over three days (19). However, the use of silver nitrate has its share of problems. It is water insoluble and susceptible to light. The solution needs to be freshly prepared and autoclaved. Evaporation of water in the autoclave may alter the concentration of the solution (8). Most patients complain of nausea, vomiting, flank pain and hematuria (4). Anaphylactic reactions, ureteric strictures and severe chemical cystitis are known to occur with its use (10). Even death has been reported following bilateral RPIS using 3\% silver nitrate (14). 
In addition, there is a significant failure rate ranging from $22-30 \%(5,6)$.

In the search to achieve a more safe and effective sclerosant various substances have been used. Povidone iodine has been recently evaluated either alone or in combination with $50 \%$ dextrose solution (7-9). It is an iodine complex with the non-ionic surfactant polymer polyvinylpyrrolidone. It is also water-soluble and releases iodine slowly. This procedure provides a non-toxic, non-irritating, non-volatile and non-staining form of iodine. It exerts a local sclerosant action and has antibacterial, antiseptic $a$ nd antifungal properties. Moreover, it is inexpensive, easily available and can be easily diluted to the required concentration Shanmugam et al. treated five patients with a single instillation of $0.2 \%$ solution prepared by diluting $5 \%$ povidone iodine with distilled water in the ratio of 1:5. All their patients were free of symptoms at six months follow-up (7). Goel et al. performed eight hourly instillations of $0.2 \%$ povidone iodine, to a total of nine doses. They first assessed the renal pelvic volume by retrograde pyelography and then accordingly instilled the sclerosing solution in volumes varying from 6-10 $\mathrm{mL}$ with the patient in Trendelenburg position. These authors had recurrence in $22 \%$ of patients and found povidone iodine as effective as silver nitrate (8). Nandy et al. used a combination of $5 \mathrm{~mL}$ povidone iodine with $5 \mathrm{~mL}$ of $50 \%$ dextrose, which was instilled twice a day with the patient in Trendelenburg position for 3 days. They had complete remission in $87 \%$, persistence in $13 \%$ and noted recurrence in 2 out of 47 patients (9). In all of these studies bilateral instillation was not performed during the same sitting.

These studies demonstrate that povidone iodine is associated with satisfactory results in the treatment of chyluria. In addition, meglumine diatrizoate (Urograffin 76\%) has been used for RPIS (10). We have studied the efficacy of a combination of 5\% povidone iodine with contrast agent (Urograffin 76\%) diluted with sterile water in a ratio of $1: 1: 3$. This ratio was arbitrarily decided and as it initially produced good results. . As fluoroscopy was used during the procedure with a contrast agent the pelviocalyceal system was well delineated. The most common site of lymphatic-urinary communication is at the fornices; hence the sclerosing solution was instilled until the blunting of all fornices was seen. Thus the chances of overlooking any fistulae were minimized. In addition, the instillation of the sclerosing solution was stopped once all the fornices were blunted. Over distension of the pelviocalyceal system, with its associated risk of pyelointerstitial and pyelo-venous backflow, was thus avoided. Shanmugam et al. used a single instillation of $8-10 \mathrm{~mL}$ of diluted povidone iodine (7). As the capacity of the pelviocalyceal system varies from each individual it is only logical that the amount of sclerosing solution needed to optimally fill the pelviocalyceal system could vary from patient to patient. Prior retrograde pyelography can determine the exact pelvic volume. Considering these facts we have combined contrast agent with povidone iodine. By using fluoroscopy we can visualize the complete filling of the system. In addition, the iodine content of the contrast agent helps to enhance the sclerosing efficacy of the solution. This is supported by the fact that good results were obtained by other investigators when contrast agents alone were used (10).

The optimum time for which sclerosing agent should be in the pelviocalyceal system is not known but it should be long enough to induce chemical lymphangitis and edema of lymphatic channels. Most of the investigators have used from three to nine instillations for RPIS $(4,5,8,9)$. In our study we instilled the sclerosing solution using a bulb tip ureteric catheter and once the system was optimally distended, blocked the end of the ureteric catheter and kept it at the ureteric orifice to prevent the sclerosing solution from effluxing out of the system. The sclerosing solution was kept in the system for five minutes. Whether keeping the solution in the collecting system for a lesser period of time will produce the desired result may be answered by a separate study.

We had a success rate of $87.5 \%$ using a single instillation. This was comparable with the results of the two other series using povidone iodine where the sclerosing solution was instilled either thee times or twice a day over a three day period. There was recurrence in only five patients who were treated again with satisfactory result. In one patient the chyluria persisted for two days and then cleared. We feel that this could have been caused by delayed occurrence of edema due to the chemical lymphangitis. The patient was free from chyluria at a follow-up of five months. The procedure was well tolerated by all patients who 
participated in the study. There was minimal pain. No major side effects or complications were observed. The procedure was done bilaterally in 14 patients in the same sitting with no side effects. The incidence of bilateral chylous efflux was higher in our study however we could find no particular explanation for this occurrence.

\section{CONCLUSION}

The authors suggest that the use of a combination of povidone iodine with contrast agent is safe and effective for the RPIS of chyluria. Fluoroscopic guidance helps to optimally fill the pelviocalyceal system. Thus under filling of the system is avoided and the chances of resultant failure of the therapy are minimized. In addition, as overfilling of the system does not occur and the complications due to pyelointerstitial and pyelo-venous backflow are prevented. This would otherwise occur if the sclerosing solution were instilled in random amounts exceeding the capacity of the pelviocalyceal system.

In this study a single instillation was as effective as multiple instillations done by other investigators. This also reduced the need for prolonged hospitalization of the patients.

A drawback of this study is the absence of a control arm and a relatively short follow up in majority of the patients. However, our results in fact suggest that this particular form of RPIS using fluoroscopy for the instillation of a combination of povidone iodine with contrast agent is safe, inexpensive, effective, minimally invasive and is associated with a short hospital stay. Also the procedure can easily and safely be reapplied in patients with recurrence.

\section{CONFLICT OF INTEREST}

None declared.

\section{REFERENCES}

1. Ohyama C, Saita H, Miyasato N: Spontaneous remission of chyluria. J Urol. 1979; 121: 316-7.
2. Date A, John TJ, Chandy KG, Rajagopalan MS, Vaska PH, Pandey AP, et al.: Abnormalities of the immune system in patients with chyluria. Br J Urol. 1981; 53: 384-6.

3. Brunkwall J, Simonsen O, Bergqvist D, Jonsson K, Bergentz SE: Chyluria treated with renal auto transplantation. A case report. J Urol. 1990; 143: 793-6.

4. Sabnis RB, Punekar SV, Desai RM, Bradoo AM, Bapat SD: Instillation of silver nitrate in the treatment of chyluria. Br J Urol. 1992; 70: 660-2.

5. Dalela D, Kumar A, Ahlawat R, Goel TC, Mishra VK, Chandra H: Routine radio-imaging in filarial chyluria-is it necessary in developing countries? Br J Urol. 1992; 69: 291-3.

6. Tan LB, Chiang CP, Huang CH, Chou YH, Wang CJ: Experiences in the treatment of chyluria in Taiwan. $\mathrm{J}$ Urol. 1990; 144: 710-3.

7. Shanmugam TV, Prakash JV, Sivashankar G: Povidone iodine used as a sclerosing agent in the treatment of chyluria. Br J Urol. 1998; 82: 587.

8. Goel S, Mandhani A, Srivastava A, Kapoor R, Gogoi $\mathrm{S}$, Kumar A, et al.: Is povidone iodine an alternative to silver nitrate for renal pelvic instillation sclerotherapy in chyluria? BJU Int. 2004; 94: 1082-5.

9. Nandy PR, Dwivedi US, Vyas N, Prasad M, Dutta B, Singh PB: Povidone iodine and dextrose solution combination sclerotherapy in chyluria. Urology. 2004; 64: 1107-9; discussion 1110.

10. Pandey AP. Chyluria. In: Morris PJ, Wood WL (eds.), Oxford Textbook of Surgery. Oxford, Oxford University Press. 2000, vol. 3, pp. 3321-3.

11. Dash SC, Bhargav Y, Saxena S, Agarwal SK, Tiwari SC, Dinda A: Acute renal failure and renal papillary necrosis following instillation of silver nitrate for treatment of chyluria. Nephrol Dial Transplant. 1996; 11: 1841-2.

12. Srivastava DN, Yadav S, Hemal AK, Berry M: Arterial haemorrhage following instillation of silver nitrate in chyluria: treatment by coil embolization. Australas Radiol. 1998; 42: 234-5.

13. Gulati MS, Sharma R, Kapoor A, Berry M: Pelvi-calyceal cast formation following silver nitrate treatment for chyluria. Australas Radiol. 1999; 43: 102-3.

14. Mandhani A, Kapoor R, Gupta RK, Rao HS: Can silver nitrate instillation for the treatment of chyluria be fatal? Br J Urol. 1998; 82: 926-7.

15. Hemal AK, Gupta NP: Retroperitoneoscopic lymphatic management of intractable chyluria. J Urol. 2002; 167: 2473-6.

16. Ciferri F, Glovsky MM: Chronic chyluria: a clinical study of 3 patients. J Urol. 1985; 133: 631-4. 
17. Norões J, Addiss D, Santos A, Medeiros Z, Coutinho A, Dreyer G: Ultrasonographic evidence of abnormal lymphatic vessels in young men with adult Wuchereria bancrofti infection in the scrotal area. J Urol. 1996; 156: 409-12.

18. Nutman TB, Kumaraswami V: Regulation of the immune response in lymphatic filariasis: perspectives on

Correspondence address:

Dr. Gyanendra Sharma

Chitale Clinic Pvt. Ltd

165 D Railway Lines

Opposite Bus Depot

Solapur, Maharashtra, 413001, India

E-mail: drgrsharma@yahoo.co.in

\section{EDITORIAL COMMENT}

The authors have instilled a mixture of povidone iodine and meglumine diatrizoate in the involved pelviocalyceal system using a bulb tipped ureteric catheter. While the collecting system is being filled it is monitored fluoroscopically to achieve a socalled 'completely full' system. The bulb of ureteric catheter is intended to keep the ureter 'completely' occluded for 'five minutes'. The authors claim that by using this methodology they prevent overfilling of system and thus the pyelointerstitial back flow and its consequences are avoided.

This claim appears to be more of a conjecture because during the period of five minutes while the ureter is occluded by bulb tipped ureteric catheter, the kidney will continue to produce urine, which, at least theoretically, may blowup the system and open up pyelointerstitial/pyelo-lymphatic backflows.

Nevertheless, it is the first report on use of povidone iodine with contrast media. To date, the problem of best dose, best concentration and no. of acute and chronic infection with Wuchereria bancrofti in South India. Parasite Immunol. 2001; 23: 389-99.

19. Dalela D, Rastogi M, Goel A, Gupta VP, Shankhwar $\mathrm{SN}$ : Silver nitrate sclerotherapy for 'clinically significant' chyluria: a prospective evaluation of duration of therapy. Urol Int. 2004; 72: 335-40.

Accepted after revision:

January 18, 2008

instillations remains vexed. Controlled studies are needed to clarify the same.

Recent reports have generated interest in the role of doxycycline as a drug to reduce the pathology of lymphatic filariasis (1). Its applicability to patients with chyluria needs to be locked into.

\section{REFERENCE}

1. Debrah AY, Mand S, Specht S, Marfo-Debrekyei Y, Batsa L, Pfarr K, et al.: Doxycycline reduces plasma VEGF-C/sVEGFR-3 and improves pathology in lymphatic filariasis. PLoS Pathog. 2006; 2: e92.

Dr. D. Dalela Department of Urology King George Medical University Lucknow, Uttar Pradesh, India E-mail:drdalela@satyam.net.in 


\section{EDITORIAL COMMENT}

Sclerotherapy, that is a minimally invasive treatment modality, is justified once conservative modalities fail. Different investigators have used many sclerosants in different concentrations. However, the maximum experience has been with silver nitrate. Because of the various problems associated with silver nitrate, recently there has been shift and many urologists have started using povidone iodine. The results of chyluria are mostly evaluated based on patient's history of any recurrence of milky urine (which may be associated with pitfalls like under or over reporting). In the study reported by Sharma et al, the authors have not mentioned the follow-up protocol. In all probability, it is also based on the patient's evidence of milky urine. It would be interesting to see if the disease also responds completely biochemically. Estimation of urinary triglycerides is considered $100 \%$ sensitive and specific test for chyluria (1). It is noninvasive and cost effective and is independent of manual error. Whether chyluria is continuous/intermittent, mild or severe, urinary triglycerides are invariably detected in morning samples (2). Estimation of urinary triglyceride levels pre- and post- instillation of sclerosants may also give insight about the patients who are likely to recur. Follow-up of patients of chyluria is extremely difficult. If we can predict recurrence based on biochemical triglyceriduria then it may help us in designing better therapy for this problem.

\section{REFERENCE}

1. Johnston DW: Chyluria: Clinical, laboratory, and statistical study of 45 personal cases observed in Hawaii. J Urol. 1945; 42: 931.

2. Dalela D: Issues in etiology and diagnosis making of chyluria. Indian J Urol. 2005; 21: 18-23.

Dr. Apul Goel Department of Urology King George Medical University Lucknow, India E-mail: goelapul1@rediffmail.com 